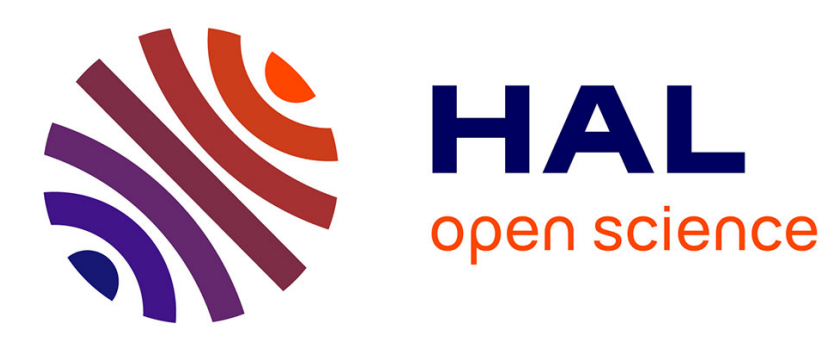

\title{
An Internal Variable Model for the Dynamic Reponse of Composites with Periodic Microstructure
}

\author{
C. Wozniak
}

\section{To cite this version:}

C. Wozniak. An Internal Variable Model for the Dynamic Reponse of Composites with Periodic Microstructure. Journal de Physique IV Proceedings, 1997, 07 (C3), pp.C3-833-C3-840. 10.1051/jp4:19973141 . jpa-00255429

\section{HAL Id: jpa-00255429 https://hal.science/jpa-00255429}

Submitted on 1 Jan 1997

HAL is a multi-disciplinary open access archive for the deposit and dissemination of scientific research documents, whether they are published or not. The documents may come from teaching and research institutions in France or abroad, or from public or private research centers.
L'archive ouverte pluridisciplinaire HAL, est destinée au dépôt et à la diffusion de documents scientifiques de niveau recherche, publiés ou non, émanant des établissements d'enseignement et de recherche français ou étrangers, des laboratoires publics ou privés. 


\title{
An Internal Variable Model for the Dynamic Reponse of Composites with Periodic Microstructure
}

\author{
C. Wozniak and M. Wozniak*
}

Institute of Mathematics and Computer Science, TU Czestochowa, Dabrowsskiego 69, PL. 42200 Czestochowa, Poland

* Department of Geotechnical and Structure Engineering (K66), TU Lódz, Al Politechniki 6, PL. 90924 Lódz 40, Poland

\begin{abstract}
A new unified micromechanical approach to dynamics of micro-periodic composite solids is formulated The proposed approach introduces the concept of internal variables in order to describe the effect of the microstructure size on the global body behaviour. It is shown that the evolution equation for internal variables can be obtained without any specification of the material properties of the composite.
\end{abstract}

\section{Introduction}

It is known that the behaviour of the composite solids with periodic microstructure can be examined on two levels. On the micro-level the interactions between constituents of a composite are detailed while the global body response is investigated in the framework of macromechanics. The passage from micro- to macromechanics is realized by so called micromechanical approaches, [1], leading to various mathematical models of the composite solid on the macrolevel. The best known from them are those based on the concept of homogeneous equivalent body where the micro-heterogeneous composite is modelled as made of a certain "homogenized" material. The above models can be obtained from some special procedures, $[1,12]$, derived by means of the asymptotic methods, $[3,5,11]$, by the Fourier expansions, [23], or using so called micro-local parameters, $[15,32]$. However, following the concept of the homogeneous equivalent body we neglect the effect of the microstructure size on the global body behaviour. This effect plays an important role mainly in the vibration and wave propagation analysis. In order to describe dynamic problems in the framework of macromechanics a number of mathematical models, mainly based on the concept of the continuum with the extra local degrees of freedom, or obtained by finding the higher-order terms of the asymptotic expansions. was proposed, $[2,9,14,15,22,24]$. Models of this kind have rather complicated analytical form and applied to the investigation of boundary-value problems often lead to the large number of boundary conditions which may be not well motivated from the physical viewpoint. Between the models using the concept of the homogeneous equivalent body and those applying continua with the extra local degrees of freedom are situated the models of the refined macrodynamics, [32]. The effect of the periodic microstructure size on the dynamic body behaviour in the framework of refined macrodynamics is described by certain unknown fields, called macro-internal variables (MIV). These variables, being governed by ordinary differential equations involving time derivatives, do not enter boundary conditions. So far, the internal variables were mainly used in formulations of the constitutive relations, [7]. Applications of this concept to the micromechanical approach to dynamics of periodic composite solids were recently investigated in a series of papers $[4,6,8,10,13,16,18-21,26-30,33-40]$.

In the aforementioned papers the micromechanical approach to macromechanio using the macro-internal variables, was based on certain heuristic assumptions related to the 
specification of materials and expected motions of the body. The aim of this contribution is to derive the governing equations for models with MIV without those assumptions. The main result is that the evolution equations for MIV can be obtained without any specification of the material properties of a composite solid. The investigations are related to composites with perfectly bonded constituents and are carried out in the framework of the small displacement gradient theory. Throughout the paper all capital Roman superscripts run over $1, \ldots, N$ (summation convention holds unless otherwise stated). Points of the physical space $\mathrm{E}$ are denoted by $\mathbf{x}, \mathbf{y}$ or $\mathbf{z}$ and their distance by $\|\mathbf{x}-\mathbf{y}\|$. The letter $t$ stands for the time coordinate and $t \in\left[t_{0}, t_{f}\right]$. By || we define both the absolute value of a real number and the length of a vector. It is assumed that all introduced functions satisfy the regularity conditions required in the subsequent analysis.

\section{Analytical preliminaries}

Let $\Omega$ be a region in the Euclidean 3-space E occupied by the composite solid in the reference configuration. Setting $\mathrm{V}:=\left(-l_{1} / 2, l_{1} / 2\right) \times\left(-l_{2} / 2, l_{2} / 2\right) \times\left(-l_{3} / 2, l_{3} / 2\right)$ we assume that the solid in this configuration has the V-periodic heterogeneous structure (is V-periodic) and that the microstructure length parameter defined by $l:=\sqrt{l_{1}^{2}+l_{2}^{2}+l_{3}^{2}}$ is negligibly small as compared to the smallest characteristic length dimension $L_{\Omega}$ of $\Omega$. We shall use the denotation $V(x)=x+V$; if $\mathrm{V}(\mathbf{x}) \subset \Omega$ then $\mathrm{V}(\mathbf{x})$ will be called the cell or the volume element of $\Omega$. The set $\Omega_{0}:=\{\mathbf{x} \in \Omega$; $\mathrm{V}(\mathbf{x}) \subset \Omega\}$ is said to be the macro-interior of $\Omega$. For an arbitrary integrable function $f(\cdot)$, defined almost everywhere on $\Omega$, we define the averaged value of $f(\cdot)$ on $V(x)$ by means of

$$
<\mathrm{f}(\mathbf{z})>(\mathbf{x})=\frac{1}{l_{1} l_{2} l_{3}} \int_{\mathrm{V}(\mathbf{x})} \mathrm{f}(\mathbf{z}) \mathrm{dv}(\mathbf{z}), \quad \mathbf{x} \in \Omega_{\mathbf{0}} .
$$

If $f(\cdot)$ is a $V$-periodic function then $\langle f(z)\rangle(x)$ is a constant which will be denoted by $\langle f\rangle$. Now we shall recall two auxiliary concepts which will be used in the subsequent analysis, [32].

Let $\Phi(\cdot)$ be a real valued function defined on $\Omega$, which represents a certain scalar field. Let us assume that the values of this field in the problem under consideration have to be calculated and/or measured up to a certain tolerance determined by the tolerance parameter $\varepsilon_{\Phi}$, $\varepsilon_{\Phi}>0$. It means that an arbitrary real number $\Phi_{0}$ satisfying condition

$$
\left|\Phi(\mathbf{x})-\Phi_{0}\right|<\varepsilon_{\Phi}
$$

can be also treated as describing with the sufficient accuracy the value of this field at the point $\mathbf{x}$. The triple $(\Phi(\cdot), \varepsilon \Phi, l)$ will be called the $\varepsilon$-macrofunction (related to the region $\Omega$ ) if the following condition holds

$$
\left(\forall(\mathbf{x}, \mathbf{y}) \in \Omega^{2}\right)[\|\mathbf{x}-\mathbf{y}\|<l \Rightarrow|\Phi(\mathbf{x})-\Phi(\mathbf{y})|<\varepsilon \Phi] .
$$

Roughly speaking, from both calculation and measurement viewpoint, every $\varepsilon$-macrofunction restricted to an arbitrary cell $\mathrm{V}(\mathbf{x}), \mathbf{x} \in \Omega_{\mathbf{0}}$, can be treated as constant.

In order to introduce the second auxiliary concept used in the subsequent analysis define by $\mathrm{h}^{\mathrm{A}}(\cdot), \mathrm{A}=1,2, \ldots$, the system of linear independent continuous $\mathrm{V}$-periodic functions (and hence defined on E) having continuous first-order derivatives. Let the above functions satisfy conditions

$$
<\mathrm{h}^{\mathrm{A}}>=\mathbf{0}, \quad<\mathrm{h}^{\mathrm{A}} \mathrm{h}^{\mathrm{B}}>=\delta^{\mathrm{AB}} l^{2}, \quad(\forall \mathbf{x})\left[\left|\mathrm{h}^{\mathrm{A}}(\mathbf{x})\right| \leq l\right]
$$

and constitute a basis in the space of sufficiently regular functions defined on an arbitrary cell $V(x)$ and having on $V(x)$ the averaged values equal to zero. We also assume that for every $h^{\wedge}(\cdot)$ there 
exist a $V$-periodic lattice $\Lambda_{A}$ of points in $E$ such that $h^{A} / \partial v(x)=0$ for every $x \in \Lambda_{A}$. It means that $\left(\forall h^{A}\right)\left((\exists x)\left[h^{A} / \partial v(x)=0\right]\right.$. Under the aforementioned conditions the system $h^{A}(\cdot), A=1,2, \ldots$, will be called the local oscillation basis.

\section{Foundations of kinematics}

Let $\mathbf{u}(\cdot, t)$ stand for a displacement field defined on $\Omega$ for every instant $t$. Define on $\Omega_{0}$ the averaged displacement field by means of

$$
\mathbf{U}(\mathbf{x}, \mathfrak{t}):=\left\langle\mathbf{u}(\mathbf{z}, \mathfrak{t})>(\mathbf{x}), \quad \mathbf{x} \in \Omega_{\mathbf{0}} .\right.
$$

By the local displacement oscillations we shall mean the vector functions $\mathbf{w}_{\mathbf{x}}(\cdot, \mathrm{t})$ defined independently on every $V(x), x \in \Omega_{\mathbf{0}}$, such that

$$
\mathbf{w}_{\mathbf{x}}(\mathbf{y}, \mathrm{t})=\mathbf{u}(\mathbf{y}, \mathrm{t})-\mathbf{U}(\mathbf{y}, \mathrm{t})+\mathbf{r}_{\mathbf{x}}(\mathbf{y}, \mathrm{t}), \quad \mathbf{y} \in \mathrm{V}(\mathbf{x}),
$$

where $\mathbf{r}_{\mathbf{x}}(\cdot)$ satisfy condition

$$
<\mathbf{r}_{\mathbf{x}}(\mathbf{z}, \mathrm{t})>(\mathbf{y})=\langle\mathbf{U}(\mathbf{z}, \mathrm{t})>(\mathbf{x})-\mathbf{U}(\mathbf{x}, \mathrm{t}),
$$

and will be specified at the end of this Section. It can be seen that

$$
<\mathbf{w}_{\mathbf{x}}(\mathrm{z}, \mathrm{t})>(\mathbf{x})=\mathbf{0}, \quad \mathrm{x} \in \Omega_{\mathbf{0}},
$$

and hence, under the known regularity conditions, every function $w_{x}(\cdot, t)$ can be represented by the Fourier series in the local oscillation basis $\mathrm{h}^{\mathrm{A}}(\cdot), \mathrm{A}=1,2, \ldots$, . Denoting the Fourier coefficients by

we obtain

$$
\mathbf{w}^{\mathrm{A}}(\mathbf{x}, \mathrm{t}):=<\mathbf{w}_{\mathbf{x}}(\mathbf{z}, \mathrm{t}) \mathrm{h}^{\mathrm{A}}(\mathbf{z})>(\mathbf{x}) l^{-2}, \quad \mathbf{x} \in \Omega_{\mathbf{0}}
$$

$$
\mathbf{w}_{\mathbf{x}}(\mathbf{y}, \mathfrak{t})=\sum_{A=1}^{\infty} \mathbf{w}^{\mathrm{A}}(\mathbf{x}, \mathfrak{t}) \mathrm{h}^{\mathrm{A}}(\mathbf{y}), \quad \mathbf{y} \in \mathrm{V}(\mathbf{x}), \quad \mathbf{x} \in \Omega_{\mathbf{0}} .
$$

The kinematics of the composites under consideration will be based on two assumptions.

Truncation Assumption (TA) states that the Fourier series (3.4) can be approximated by the sum of the first $\mathrm{N}$ terms for some $\mathrm{N} \geq 1$, where $\mathrm{N}$ has to be specified in every problem under consideration.

From TA it follows that instead of (3.4) we assume

where here and in the sequel the Roman superscripts run over $1, \ldots, N$ (summation convention holds). The functions $\mathrm{h}^{\mathrm{A}}(\cdot), \mathrm{A}=1,2, \ldots, \mathrm{N}$ are called the micro-shape functions.

Kinematic Macro-Regularity Assumption (KRA) restricts the class of motions in every problem under consideration by assuming that fields $U(\cdot, t), W^{A}(\cdot, t), A=1,2, \ldots, N$, together with their derivatives, are regular $\varepsilon$-macrofunctions.

It can be seen that the formulation of KRA takes into account TA by means of which the number $\mathrm{N}$ of the micro-shape function is postulated in every problem under consideration. Under KRA fields $\mathbf{U}(\cdot, \mathrm{t}), \mathbf{W}^{A}(\cdot, \mathrm{t}), \mathrm{A}=1,2, \ldots, \mathrm{N}$, are said to be the macrodisplacements and the macro-internal variables (MIV), respectively. The meaning of the term MIV will be explained in Sec. 5. The $\varepsilon$-macrofunctions $\mathbf{U}(\cdot, \mathrm{t}), \mathbf{W}^{A}(\cdot, \mathrm{t})$ describe the kinematics of composites on the macro-level (macro-kinematics) and will constitute the basic kinematic unknowns in the framework of the proposed model. The results of this section are summarized by the following lemma. 
Lemma. Under TA the displacements on the micro-level are related to the macrodisplacements and the macro-internal variables by the formulae

$$
\mathbf{u}(\mathbf{y}, t)=\mathbf{U}(\mathbf{y}, \mathbf{t})+\mathrm{h}^{\mathrm{A}}(\mathbf{y}) \mathbf{W}^{\mathrm{A}}(\mathbf{y}, \mathrm{t}) \text {. }
$$

The proof of the above lemma is based on the specification of fields $\mathbf{r}_{\mathbf{x}}(\cdot, \mathrm{t})$ to the form

$$
\mathbf{r}_{\mathbf{x}}(\mathbf{y}, \mathrm{t})=\mathrm{h}^{\mathrm{A}}(\mathbf{y})\left[\mathbf{w}^{\mathrm{A}}(\mathbf{x}, \mathrm{t})-\mathbf{W}^{\mathrm{A}}(\mathbf{y}, \mathrm{t})\right], \quad \mathrm{y} \in \mathrm{V}(\mathbf{x}), \quad \mathrm{A}=1, \ldots, \mathrm{N} .
$$

Substituting the right-hand sides of (3.7) into (3.2) and using (3.5) we arrive at (3.6).

\section{From micro- to macrodynamics}

Let $\mathbf{s}(\cdot, \mathbf{t})$ denote the Cauchy stress tensor field defined for every $t$ on $\Omega \backslash \Gamma$ where $\Gamma$ is a set of all interfaces between the components of the composite. Let us define on $\Omega_{0}$ the following averaged stress fields

(4.1) $\mathbf{S}(\mathbf{x}, \mathrm{t}):=<\mathbf{s}(\mathbf{z}, \mathrm{t})>(\mathbf{x}), \quad \mathbf{H}^{\mathrm{A}}(\mathbf{x}, \mathrm{t}):=<\mathbf{s}(\mathbf{z}, \mathrm{t}) \cdot \nabla \mathbf{h}^{\mathrm{A}}(\mathbf{z})>(\mathbf{x}), \quad \mathrm{x} \in \Omega_{\mathbf{0}}$.

In order to pass from micro- to macrodynamics two extra assumptions will be required.

Stress Macro-Regularity Assumption (SRA) restricts the class of stress fields in the problem under consideration to that in which the fields $\mathbf{S}(\cdot, \mathrm{t}), \mathbf{H}^{\mathbf{A}}(\cdot, \mathrm{t})$ together with their derivatives are regular $\varepsilon$-macrofunctions.

Under SRA the fields defined by (4.1) will be called the macrostresses and the microdynamic forces, respectively. The meaning of the second from these terms will be explained at the end of this section.

Let $\rho(\cdot)$ stand for the mass density field (which is the V-periodic function defined almost everywhere on $\Omega$ ) and assume that the body force $\mathbf{b}$ is constant. Let us denote by $\mathbf{n}(\mathbf{y})$ the unit normal outward to $\partial \mathrm{V}(\mathbf{x})$ at $\mathbf{y}$. The starting point of the proposed micromechanical procedure will be the weak form of equations of motion in micromechanics. Taking into account the symmetry of the stress tensor these equations can be assumed in the form of conditions

$$
\int_{V(\mathbf{x})} \mathbf{s}(\mathbf{y}, \mathrm{t}): \nabla \overline{\mathbf{u}}(\mathbf{y}) \mathrm{dv}=\oint_{\partial \mathrm{V}(\mathbf{x})}[\mathbf{s}(\mathbf{y}, \mathbf{t}) \cdot \mathbf{n}(\mathbf{y})] \cdot \overline{\mathbf{u}}(\mathbf{y}) \mathrm{da}+\int_{\mathrm{V}(\mathbf{x})} \rho(\mathbf{y})[\mathbf{b}-\ddot{\mathbf{u}}(\mathbf{y}, \mathbf{t})] \cdot \overline{\mathbf{u}}(\mathbf{y}) \mathrm{dv}
$$

which have to hold for every $x \in \Omega_{0}$ and for an arbitrary test function $\mathbf{u}(\cdot)$. In order to pass from micro- to macrodynamics we have to specify the set of test functions in (4.2). Taking into account (3.6) we assume that

$$
\overline{\mathbf{u}}(\mathbf{y})=\overline{\mathbf{U}}(\mathbf{y})+\mathrm{h}^{\mathrm{A}}(\mathbf{y}) \overline{\mathbf{W}}^{\mathrm{A}}(\mathbf{y}),
$$

where $\overline{\mathbf{U}}(\cdot), \overline{\mathbf{W}}^{\mathrm{A}}(\cdot)$ are arbitrary linear independent regular $\varepsilon$-macrofunctions defined on $\Omega$.

Now we shall formulate the fundamental assertion of the micromechanical approach to macrodynamics proposed in this contribution. For the sake of simplicity we shall also assume that the micro-shape functions $\mathrm{h}^{\mathrm{A}}(\cdot)$ satisfy the extra conditions $\left\langle\rho \mathrm{h}{ }^{\mathrm{A}}>=0, \mathrm{~A}=1, \ldots, \mathrm{N}\right.$.

Fundamental Assertion. Under TA, KRA, SRA and in the framework of MAA the equations of motion (4.2) imply the following interrelation between the macrodisplacements $\mathbf{U}(\cdot, \mathrm{t})$ and the macrostresses $\mathbf{S}(\cdot, \mathrm{t})$ :

$$
\operatorname{Div} \mathbf{S}(\mathbf{x}, \mathrm{t})-<\rho>\ddot{\mathbf{U}}(\mathbf{x}, \mathrm{t})+<\rho>\mathbf{b}=\mathbf{0},
$$

as well as between the macro-internal parameters $\mathbf{W}^{A}(\cdot, t)$ and the micro-dynamic forces $\mathbf{H}^{A}(\cdot, t)$ :

$$
<\rho \mathrm{A}^{\mathrm{A}} \mathrm{h}^{\mathrm{B}}>\ddot{\mathbf{W}}^{\mathrm{B}}(\mathbf{x}, \mathrm{t})+\mathbf{H}^{\mathrm{A}}(\mathbf{x}, \mathrm{t})=\mathbf{0}, \quad \mathrm{A}=1, \ldots, \mathrm{N} ;
$$

the above relations hold for every $x \in \Omega_{0}$ and $t \in\left(t_{0}, t_{f}\right)$.

The relations given by (4.4), (4.5) will be called the equations of motion and the dynamic evolution equations, respectively. Since the modulae $\left\langle\rho \mathrm{A}^{\mathrm{A}} \mathrm{h}^{\mathrm{B}}\right\rangle$ are of an order $O\left(l^{2}\right)$ then the 
above equations describe the effect of the microstructure size $l$ on the body dynamic response. For this reason equations (4.4), (4.5) are said to represent the refined macrodynamics of the composites under consideration, [30]. Let us observe that both in the quasi-stationary processes and for problems in which the above effect can be neglected we obtain $\mathbf{H}^{\mathbf{A}}(\mathbf{x}, \mathbf{t})=\mathbf{0}$. That is why the fields defined by the second from Eqs (4.1) were called the micro-dynamic forces. It has to be emphasized that under aforementioned assumptions equations (4.4), (4.5) are related to a composite solid with periodic microstructure made of arbitrary materials. Hence, the aforementioned equations represent the averaged laws of motion in the framework of the proposed refined macrodynamics. The proof of fundamental assertion can be found in [38].

\section{MIV-model}

In order to describe the dynamic response of the composite body in the framework of the MIVmodel we have to complete equations (4.4), (4.5) introducing the constitutive equations for macrostresses $\mathbf{S}$ and micro-dynamic forces $\mathbf{H}^{\mathrm{A}}$. Taking into account the definitions (4.1), the second from the formulae (3.7) and applying MAA to the integrals in (4.1), this can be done for arbitrary periodic composites the components of which are simple materials. To simplify the subsequent considerations we shall restrict ourselves to the linear visco-elastic materials governed by the constitutive equations of the form

$$
\mathbf{s}=\mathbf{C}(\mathbf{z}): \mathbf{e}+\mathbf{D}(\mathbf{z}): \dot{\mathbf{e}} ; \quad \mathbf{e}:=0.5\left[\nabla \mathbf{u}+(\nabla \mathbf{u})^{\mathbf{T}}\right],
$$

where $\mathbf{C}(\cdot), \mathbf{D}(\cdot)$ are $\mathrm{V}$-periodic piecewise constant functions the values of which are the fourth order tensors of elastic and viscous modulae, respectively, for the component materials. Define the linearized macro-strain tensor by means of

$$
\mathbf{E}(\mathbf{x}, \mathbf{t}):=0.5\left[\nabla \mathbf{U}(\mathbf{x}, \mathbf{t})+(\nabla \mathbf{U}(\mathbf{x}, \mathbf{t}))^{\mathbf{T}}\right] .
$$

Substituting (5.1) into definitions (4.1) and using MAA we obtain

$$
\begin{aligned}
& \mathbf{S}(\mathbf{x}, \mathrm{t})=\left\langle\mathbf{C}>: \mathbf{E}(\mathbf{x}, \mathrm{t})+<\mathbf{C} \cdot \nabla \mathrm{h}^{\mathrm{A}}>\cdot \mathbf{W}^{\mathrm{A}}(\mathbf{x}, \mathrm{t})+<\mathbf{D}>: \dot{\mathrm{E}}(\mathbf{x}, \mathrm{t})+<\mathbf{D} \cdot \nabla \mathrm{h}^{\mathrm{A}}>\cdot \dot{\mathbf{W}}^{\mathrm{A}}(\mathbf{x}, \mathrm{t}),\right. \\
& \mathbf{H}^{\mathrm{A}}(\mathbf{x}, \mathrm{t})=<\nabla \mathrm{h}^{\mathrm{A}} \cdot \mathbf{C}>: \mathbf{E}(\mathbf{x}, \mathrm{t})+<\nabla \mathrm{h}^{\mathrm{A}} \cdot \mathbf{C} \cdot \nabla \mathrm{h}^{\mathrm{B}}>\cdot \mathbf{W}^{\mathrm{B}}(\mathbf{x}, \mathrm{t})+ \\
&+<\nabla \mathrm{h}^{\mathrm{A}} \cdot \mathbf{D}>: \dot{\mathrm{E}}(\mathbf{x}, \mathrm{t})+<\nabla \mathrm{h}^{\mathrm{A}} \cdot \mathbf{D} \cdot \nabla \mathrm{h}^{\mathrm{B}}>\cdot \dot{\mathbf{W}}^{\mathrm{B}}(\mathbf{x}, \mathrm{t}),
\end{aligned}
$$

for every $x \in \Omega_{0}$ and $t \in\left(t_{0}, t_{f}\right)$. The above equations will be referred to as the macro-constitutive equations for the linear visco-elastic composites.

Equations (4.4), (4.5) and (5.2), (5.3) represent the macro-internal variable model (MTVmodel) of micro-periodic composites made of perfectly bonded visco-elastic constituents. For the linear elastic materials the above equations reduce to those of the refined macromechanics, which were obtained independently in [30] by means of certain heuristic hypotheses. For every micro-periodic composite solids (with constituents modelled as simple materials) the proposed model is uniquely determined by the choice of the micro-shape functions $h^{A}(\cdot), A=1, \ldots, N$.

It has to be emphasized that for every class of motions specified by conditions (3.6) we obtain the pertinent MIV-model. In the analysis of special problems we have to take into account only these classes of motions which seem to be relevant from the viewpoint of the engineering applications of the theory.

Substituting the right-hand sides of Eqs (5.3) into (4.4), (4.5) we obtain the system of three partial differential equations for the macrodisplacements $\mathbf{U}$ coupled with the system of $3 \mathrm{~N}$ ordinary differential equations for the macro-internal variables $\mathbf{w}^{\mathrm{A}}$. Hence, in formulations of the initial-boundary value problems, unknowns $\mathbf{W}^{\mathrm{A}}(\cdot, \mathrm{t})$ do not enter the boundary conditions. That is why they were called the macro-internal variables (MTV). It can be shown that for 
homogeneous bodies and homogeneous initial conditions for MIV, we obtain the trivial solution $W^{A}=0, A=1, \ldots, N$, to every boundary value problem. Hence, the macro-internal variables play a crucial role in a description of the dynamic behaviour of solids with periodic microstructure and that is why the models proposed were referred to as the macro-internal variable models. It has to be emphasized that solutions to special problems in the framework of MIV-models have the physical sense only if the fields $\mathbf{U}(\cdot, t), \mathbf{W}^{\mathrm{A}}(\cdot, \mathrm{t})$ as well as $\mathbf{S}(\cdot, \mathrm{t}), \mathbf{H}^{\mathbf{A}}(\cdot, \mathrm{t})$, for every instant $\mathrm{t}$, are the regular $\varepsilon$-macrofunctions. This requirement can be verified only $a$ posteriori.

\section{Conclusions}

Let us summarize the advantages and drawbacks of the MIV-models of composites in the light of their possible applications to dynamics of composite solids. The main advantages can be listed as follows.

1. The MIV-models describe the effect of the microstructure size on the dynamic behaviour of a composite body contrary to models based on the concepts of the homogeneous equivalent body. Hence, using these models we can investigate dispersion phenomena and determine higher wave propagation speeds and free vibration frequencies in composite materials. It can be observed that the MIV-models describe the length-scale effect on the composite body behaviour only in dynamic problems.

2. The form of the governing equations of the MIV-models is relatively simple since all macro-internal variables as the extra unknowns are governed by the ordinary differential equations, involving only time derivatives of $\mathbf{W}^{\mathrm{A}}$. Hence, the boundary conditions for the MIVmodels have the form similar to that met in solid mechanics. It has to be noticed that in the micromorphic models of composites, based on the concept of the extra local degrees of freedom (like the Cosserat-type continua), we deal with the large number of boundary conditions which may be not well motivated from the physical or engineering viewpoint. The same situation also holds for the asymptotic models involving higher-order approximations; this problem will be analyzed in a separate paper.

3. The governing equations of an arbitrary MIV-model have constant coefficients which can be easily determined by calculations the integrals over $\mathrm{V}$ and do not require any previous solution to the boundary value problem on the unit cell contrary to models obtained via the asymptotic methods.

4. The MIV-models have a wide scope of applications since they can be postulated in the unified way for composites made of arbitrary simple materials. Moreover, the formal procedure presented in this contribution can be easily generalized to include the problem of finite deformations.

5. In some special problems the MTV-models have an adaptive character similar to that of the FEM. It means that they can be formulated on different levels of accuracy either by applying different truncations of the Fourier series or by changing the form of micro-shape functions.

Among the drawbacks of the MIV-models the following ones seem to be the most relevant:

1. The analysis of the microdynamic effects is confined almost exclusively to the behaviour of a composite on a macro-level. The passage to microdynamics by using formulae (3.6) may require a very large number $\mathrm{N}$ of the micro-shape functions, which makes the problem very difficult to solve.

2. The choice of the Fourier expansion of local oscillations and its truncation leading to the proper MIV-models for the problem under consideration is not specified by the proposed approach. For some special problems (e.g. for laminated structures) the choice of the micro- 
shape functions can be based on the intuition of the researcher as a certain a priori postulated kinematic hypothesis not related to the aforementioned Fourier expansion.

3. Every MIV-model is restricted only to the analysis of a special class of motions which from a qualitative viewpoint has to be postulated a priori by the choice of the micro-shape functions. Hence the above models can be applied mostly to problems in which we are interested in a dynamic body behaviour under motions which can be assumed a priori as relevant for the problem under consideration.

Summarizing the above conclusions and taking into account the recently obtained results on this field (cf. the references mentioned in Introduction) one can suppose that the MIV-models of composite material structures deserve a certain attention both from the theoretical and engineering point of view.

This research work was supported by KBN Warsaw Poland under grant No. 7 T07A 01711

\section{References}

1. J. Aboudi, Mechanics of composite materials: a unified micromechanical approach, Elsevier, Amsterdam, 1991

2. J. D. Achenbach, G. Hermann, Wave motions in solids with laminar structuring, "Dynamics of Structured Solids" (ed. G. Hermann), Am. Soc. Mech. Eng., New York, 1968

3. N. S. Bakhvalov, G. P. Panasenko, Osrednienie processov v periodiceskich sredach, Nauka, Moskva, 1984

4. E. Baron, C. Woźniak, On the microdynamics of composite plates, Arch. Appl. Mech., 66, $1995,126-133$

5. A. Bensoussan, J. L. Lions, G. Papanicolaou, Asymptotic analysis for periodic structures, North- Holland, Amsterdam, 1978

6. I. Cielecka, On the continuum modelling the dynamic behaviour of certain composite latticetype structures, J. Theor. Appl. Mech., 33, 1995, 351-360

7. B. D. Coleman, M. E. Gurtin, Thermodynamics with internal state variables, J. Chem. Phys., 47,1967, 597-613

8. F. Dell'Isola, L. Rosa, C. Woźniak, Dynamics of solids with micro-periodic non-connected fluid inclusions, Arch. Appl. Mech., in the course of publication

9. G. A. Hegemier, On a theory of interacting continua for wave propagation in composites, "Dynamics of Composite Materials" (ed. E. H. Lee), Am. Soc. Mech. Eng., New York, 1972

10. J. Jędrysiak, C. Woźniak, On the elastodynamics of thin microperiodic plates, J. Theor. Appl. Mech., 33, 1995, 337-350

11. V.V. Jikov, S.M. Kozlov, O.A. Oleinik, Homogenization of differential operators and integral functionals, Springer -Verlag, Berlin, 1994

12. R. Jones, Mechanics of composite materials, Mc Graw-Hill, New York, 1972

13. S. Konieczny, M. Woźniak, On the wave propagation in fibre-reinforced composites, J. Theor. Appl. Mech., 33,1995, 375-384

14. T. Lewinski, St. Kucharski, A model with length scales for composites with periodic structure, Part I., Comp. Mechanics, 9, 1992, 249-265

15. A. Maewal, Construction of models of dispersive elastodynamic behaviour of periodic composites: a computational approach, Comp. Meth. Appl. Mech. Eng., 57, 1986, 191

16. S. J. Matysiak, W. Nagórko, On the wave propagation in periodically laminated composites, Bull. Pol. Ac. Sci.: Tech. Sci., 43, 1995, 1-12 
17. S.J. Matysiak, C. Woźniak, Micromorphic effects in a modelling of periodic multilayered elastic composites. Int. J. Engng Sci., 25, 1987, 549-551

18. K. Mazur-Sniady, Macro-dynamics of micro-periodic elastic beams, J. Theor. Appl. Mech., 31, 1993, 781-793

19. B. Michalak, C. Woźniak, M. Woźniak, The dynamic modelling of elastic wavy-plates, Arch. Appl. Mech., 66, 1996, 177-186

20. G. Mielczarek, C. Woźniak, On the dynamic modelling of fibrous composites, J. Tech. Phys, 36, 1995, 103-111

21. G. Mielczarek, C. Woźniak, On the micro-dynamics of composite materials, J. Theor. Appl. Mech., 33, 1995, 731-745

22. R. D. Mindlin, Microstnucture in linear elasticity, Arch. Rat. Mech. Anal., 16, 1964, 51-78

23. S. Nemat-Nasser, M. Hori, Micromechanics: overall properties of heterogeneous materials, Noth-Holland, Amsterdam, 1993

24. C. T. Sun, J. D. Achenbach, G. Hermann, Time-harmonic waves in a stratified medium propagating in the direction of the layering, J. Appl. Mech., 35, 1968, 408-411

25. H. F. Tiersten, M. Jahanmir, The theory of composites modelled as interpenetrating solid continua, Arch. Rat. Mech. Anal., 65, 1977, 153-192

26. M. Wagrowska, C. Woźniak, Macro-modelling of dynamic problems for visco-elastic composite materials, Int. J. Engng. Sci., vol. 34, 1996, 923-932

27. E. Wierzbicki, On the wave propagation in micro-periodic elastic media, Bull. Ac. Pol. Sci.: Tech. Sci., 41, 1993, 323-327

28. E. Wierzbicki, Nonlinear macro-micro dynamics of laminated structures, J. Theor. Appl. Mech., 33, 1995, 291-307

29. E. Wierzbicki, C. Woźniak, M. Woźniak, Finite rotations in the refined macrodynamics of elastic composites, J. Theor. Appl. Mech., 33, 1995, 15-25

30. E. Wierzbicki, C. Woźniak, M. Woźniak, Thermal stresses in nonstationary problems for composite materials, Int. J. Engng. Sci., in the course of publication

31. C. Woźniak, A nonstandard method of modelling of thermo-elastic periodic composites, Int. J. Engng Sci., 25,1987, 133-141

32. C. Woźniak, Refined macrodynamics of periodic structures, Arch. Mech., 45, 1993, 295-304

33. C. Woźniak, Micro-macro dynamics of periodic material structures, in: Structural Dynamics, Eurodyn'93, Balkema, Rotterdam, 1993, 573-575

34. C. Woźniak, Nonlinear macro-elastodynamics of microperiodic composites, Bull. Ac. Pol. Sci.: Tech. Sci., 41, 1993, 315-321

35. C. Woźniak, Microdynamics: continuum modelling the simple composite materials, $\mathrm{J}$. Theor. Appl. Mech., 33, 1995, 267-289

36. C. Woźniak, Z. F. Baczyński, M. Woźniak, Modelling of nonstationary heat conduction problems in micro-periodic composites, ZAMM, 76, 1996, 223-229

37. C. Woźniak, M. Woźniak, On the micro-modelling of dynamic response for thermoelastic periodic composites, in: Proc. IUTAM Symp. "Microstructure Property Interactions in Composite Materials", Aalborg, 1994, 387-395

38. C. Woźniak, Internal variables in dynamics of composite solids with periodic microstruture, Arch. Mech., 49, 1997, to appear

39. M. Woźniak, On the dynamic behaviour of micro-damaged stratified media, Int. J. Fract., 73, 1995, 223-232

40. M. Woźniak, 2D dynamics of a stratified elastic subsoil layer, Arch. Appl. Mech., 66, 1996, 284-290 